POS PROCEEDINGS

\title{
The TOROS project and the optical follow-up of gravitational wave events
}

\author{
Mario C. Díaz ${ }^{* \dagger}$ \\ The University of Texas Rio Grande Valley \\ E-mail: mario.diazeutrgv.edu
}

\begin{abstract}
In this short paper we describe the TOROS collaboration, its participation in the electromagnetic follow-up efforts of the LIGO VIRGO $\mathrm{O} 1$ and $\mathrm{O} 2$ observational campaigns and some its main characteristics and goals. We discuss TOROS plans for the projected O3 campaign as well.
\end{abstract}

XII Multifrequency Behaviour of High Energy Cosmic Sources Workshop

12-17 June, 2017

Palermo, Italy

${ }^{*}$ Speaker.

${ }^{\dagger}$ On behalf of the TOROS Collaboration. 


\section{Introduction}

The network of advanced ground-based gravitational-wave (GW) interferometers constituted by the LIGO observatories [12], started a second observational campaign (O2) on August 1, 2016 (the first one -O1- concluded on January 2016). These observatories were joined by the Virgo observatory [7] on August 1, 2017, initiating for the first time the concurrent observation of the sky by a network of three interferometers, which ended on August 25, 2017 ${ }^{1}$. The first gravitational wave detection GW150914 announced by the LIGO Scientific Collaboration on February 11, 2016 [1] opens the era of gravitational wave astronomy. Several more detections of Binary Black Hole mergers have been reported afterwards [4] where the second detection took place on December 26, 2015, the third one on January 4, 2017 [3], a fourth one on June 8, 2017 [5] and a fifth one, which is the first triple detector detection on August 14, 2017 [6]. This latter one confirmed prior to the June 8 detection, is the first detection made using a network of three interferometers. The detected waves -observed on August 14th, 2017 at 10:30:43 UTC (6:30AM EDT)- were produced by a pair of black holes with 31 and 25 solar masses. They merged to produce a spinning black hole of 53 solar masses. Combining the signal from Virgo with the signal observed in the two LIGO observatories improved the sky localization of the source by over a factor of 10 .

Additionally to the merger of binary black-holes, the coalescence and merger of a neutron-star - neutron-star (BNS) or neutron - star - black-hole (NS-BH) binary is among the most energetic events in the Universe expected to have an associate electromagnetic, including optical emission. This expectation was largely rewarded by the astonishing first simultaneous multi-messenger astronomy detection of such a system [2], [13]. On the 17th August 2017 at 12:41:04 UTC a binary neutron star coalescence candidate was identified in data from the LIGO Hanford observatory and later confirmed by detections from the LIGO Livingston and the VIRGO detector. The multimessenger observational effort which covered the entire electromagnetic spectrum as well as particle detectors observations was reported in [13].

Precisely to foster collaborations that could elicit such multi-messenger observations the LSC and the Virgo Collaboration (VC) issued on 2013 June 6 a worldwide call ${ }^{2}$ to participate in electromagnetic (EM) and multi-messenger observations of GW events recorded by their detectors, using a wide range of telescopes and instruments of "mainstream astronomy".

\section{The TOROS collaboration in $\mathrm{O1}$ and $\mathrm{O2}$}

The TOROS "Transient Optical Robotic Observatory of the South"3 [9], [8], was organized in 2013 to participate in these observations. The TOROS collaboration succeeded in participating in the electromagnetic follow-up effort ensuing the first detection [10]. It also participated in the $\mathrm{O} 2$ LVC observational campaign and obtained relevant photometry for the kilonova associated with GW170817 (the binary neutron star merger detected by the LIGO and VIRGO interferometers on August 17 of last year) [11], [13]. One key problem in optical following-up efforts is the following: GW detectors are all-sky detectors that allow localization via triangulation. They generally have

\footnotetext{
${ }^{1}$ http://www.ligo.org/news/index.php\#O2end

${ }^{2} \mathrm{http} / / /$ www.ligo.org/scientists/GWEMalerts.php

${ }^{3} \mathrm{http}: / /$ toros.utrgv.edu
} 
poor pointing accuracy even for high signal-to-noise ratio events, leading to uncertainties of up to dozens of square degrees in the target area for electromagnetic followup, when three detectors participate in the network (i.e. in the case of GW170817, the source was initially localized to a sky region of $31 \mathrm{deg}^{2}$ [13]). When less than three interferometers are involved in the detection the probability maps can span hundreds of square degrees. In both situations, wide-field cameras and rapid follow-up observations will be crucial for EM counterpart detections. High quality, high resolution and deep wide-field images are other crucial ingredients, which means that facilities with telescopes dedicated to the EM follow-up should be constructed at optimal locations.

\section{Conclusion: The TOROS collaboration in the future}

In addition to the general scientific motivation outlined in the previous section, we were driven by the limited number of southern facilities with wide fields of view that would be capable of dedicated searches for aLIGO and adVIRGO released triggers during their first years of operation. While TOROS has been utilizing other resources and participated in both the $\mathrm{O} 1$ and $\mathrm{O} 2$ observational campaigns as it was mentioned in the previous section it expects to advance its original project near completion this year in preparation of the $\mathrm{O} 3 \mathrm{LVC}$ observational campaign. In the remaining of this section the main characteristics of the TOROS original project will be described.

The TOROS collaboration decided that Cordón Macón, a mountain range in the province of Salta, Argentina (W67 $19^{\prime} 41.6^{\prime \prime}, \mathrm{S} 24^{\circ} 37^{\prime} 21.9^{\prime \prime}, 4637 \mathrm{~m}$ elev.) offered a unique opportunity for the development of a new astronomical facility. This site was first considered as a location to develop an astronomical facility by the European Southern Observatory, as part of their search for suitable locations for the Extremely Large European Telescope (E-ELT) ${ }^{4}$. The site, Cordón Macón, in the Atacama Plateau of northwestern Argentina was characterized during campaigns sustained in the years 2007 through 2009 ([14]. Satellite surveys also provided an assessment of the site appropriateness for astronomical observations [15].

As per design the TOROS telescope will have a primary mirror diameter of $0.6 \mathrm{~m}$, a fieldof-view of 9.85 sq.deg. and a very broad bandpass $(0.4-0.9 \mu \mathrm{m}$, equivalent to a combination of the Sloan griz filters). It will be a fully robotic facility, driven by a priority-based intelligent agent/scheduler with four modes of operation in decreasing order of priority: (1) follow up of gravitational-wave triggers; (2) follow up of $\gamma$-ray burst triggers from Fermi, Swift and other missions; (3) baseline imaging of the entire surveyable area; (4) search for short-duration transient events, variable sources and moving objects within the DES and VISTA-VIKING fields.

TOROS plans to obtain 15-minute exposures so to fully cover the median localization area for the three gravitational wave detectors scenario in just 3 to 4 sweeps, obtaining a sufficient SNR for the EM counterpart of a GW event at the median distance $(I=21.7$ mag) expecting $4-5 \sigma$ detections at peak magnitude under grey/dark sky conditions, improving to $7-9 \sigma$ for the top quartile of events $(I=21 \mathrm{mag})$. TOROS plans to promptly release coordinates of potential transients and stacked images to the entire astronomical community. Additionally, it plans to execute its own photometric and spectroscopic follow-up.

\footnotetext{
${ }^{4}$ http://www.eso.org/public/teles-instr/e-elt/
} 


\section{References}

[1] B.P.Abbott, et al. Observation of Gravitational Waves from a Binary Black Hole Merger. Phys. Rev. Lett., 116(6):061102, February 2016.

[2] B.P.Abbott, et al. GW170817: Observation of Gravitational Waves from a Binary Neutron Star Inspiral. Phys. Rev. Lett., 119, 161101, October 2017.

[3] B.P.Abbott, et al. GW170104: Observation of a 50-Solar-Mass Binary Black Hole Coalescence at Redshift 0.2. Phys. Rev. Lett., 118, 221101, June 2017.

[4] B. P. Abbott, R. Abbott, T. D. Abbott, M. R. Abernathy, F. Acernese, K. Ackley, C. Adams, T. Adams, P. Addesso, R. X. Adhikari, and et al. GW151226: Observation of Gravitational Waves from a 22-Solar-Mass Binary Black Hole Coalescence. Physical Review Letters, 116(24):241103, June 2016.

[5] B. P. Abbott, et al. GW170608: OBSERVATION OF A 19-SOLAR-MASS BINARY BLACK HOLE COALESCENCE. ApJL, 851:L35, 2017.

[6] B. P. Abbott, et al. GW170814: A Three-Detector Observation of Gravitational Waves from a Binary Black Hole Coalescence. Physical Review Letters, 119:141101 , November 2017.

[7] F. Acernese, M. Agathos, K. Agatsuma, D. Aisa, N. Allemandou, A. Allocca, J. Amarni, P. Astone, G. Balestri, G. Ballardin, and et al. Advanced Virgo: a second-generation interferometric gravitational wave detector. Classical and Quantum Gravity, 32(2):024001, January 2015.

[8] M. Benacquista, C. Belczynski, M. Beroiz, M. Branchesi, C. Colazo, M. C. Diaz, M. Dominguez, D. Garcia Lambas, S. Liang, L. Macri, T. Peñuela, B. Sanchez, M. Schneiter, and C. V. Torres. A program for optical observations of advanced LIGO early triggers in the southern hemisphere. In EAS Publications Series, volume 67 , pages 357-358, July 2014.

[9] M.C. Díaz, The TOROS project In The Third Hot-wiring the Transient Universe Workshop, ed. P. R. Wozniak et al. (Menlo Park, CA: SLAC), 225, http://www.slac.stanford.edu/econf/C131113.1/papers/diaz.pdf

[10] M. C. Díaz, M. Beroiz, T. Peñuela, L. M. Macri, R. J. Oelkers, W. Yuan, D. García Lambas, J. Cabral, C. Colazo, M. Domínguez, B. Sánchez, and S. Gurovich. GW150914: First Search for the Electromagnetic Counterpart of a Gravitational-wave Event by the TOROS Collaboration. ApJL, 828:L16, September 2016.

[11] M. C. Díaz, L. M. Macri, et al.. Observations of the First Electromagnetic Counterpart to a Gravitational-wave Source by the TOROS Collaboration. ApJL, 848:L29 (5pp), October 2017.

[12] LIGO Scientific Collaboration, J. Aasi, B. P. Abbott, R. Abbott, T. Abbott, M. R. Abernathy, K. Ackley, C. Adams, T. Adams, P. Addesso, and et al. Advanced LIGO. Classical and Quantum Gravity, 32(7):074001, April 2015.

[13] LIGO Scientific Collaboration and Virgo Collaboration, Fermi GBM, INTEGRAL, IceCube Collaboration, AstroSat Cadmium Zinc Telluride Imager Team, IPN Collaboration, The Insight-Hxmt Collaboration, ANTARES Collaboration, The Swift Collaboration, AGILE Team, The 1M2H Team, The Dark Energy Camera GW-EM Collaboration and the DES Collaboration, The DLT40 Collaboration, GRAWITA: GRAvitational Wave Inaf TeAm, The Fermi Large Area Telescope Collaboration, ATCA: Australia Telescope Compact Array, ASKAP: Australian SKA Pathfinder, Las Cumbres Observatory Group, OzGrav, DWF (Deeper, Wider, Faster Program), AST3, and CAASTRO Collaborations, The VINROUGE Collaboration, MASTER Collaboration, J-GEM, GROWTH, JAGWAR, Caltech- NRAO, TTU-NRAO, and NuSTAR Collaborations, Pan-STARRS, The MAXI 
Team, TZAC Consortium, KU Collaboration, Nordic Optical Telescope, ePESSTO, GROND, SALT Group, TOROS: Transient Robotic Observatory of the South Collaboration, The BOOTES Collaboration, MWA: Murchison Widefield Array, The CALET Collaboration, IKI-GW Follow-up Collaboration, H.E.S.S. Collaboration, LOFAR Collaboration, LWA: Long Wavelength Array, HAWC Collaboration, The Pierre Auger Collaboration, ALMA Collaboration, Euro VLBI Team, Pi of the Sky Collaboration, The Chandra Team at McGill University, DFN: Desert Fireball Network, ATLAS, High Time Resolution Universe Survey, RIMAS and RATIR, and SKA South Africa/MeerKAT. Multi-messenger Observations of a Binary Neutron Star Merger. ApJL, 848:L12, October 2017.

[14] V. Renzi, R. Vrech, D. Ferreiro, D. García Lambas, M. Solinas, H. Muriel, J. Viramonte, M. Sarazin, and P. Recabarren. Caracterización astronómica del sitio Cordón Macón en la provincia de Salta. Boletin de la Asociacion Argentina de Astronomia La Plata Argentina, 52:285-288, 2009.

[15] P. Tremblin, N. Schneider, V. Minier, G. A. Durand, and J. Urban. Worldwide site comparison for submillimetre astronomy. AAP, 548:A65, December 2012. 